\title{
The TALKS study to improve communication, logistical, and financial barriers to live donor kidney transplantation in African Americans: protocol of a randomized clinical trial
}

\author{
Tara S. Strigo ${ }^{1}$, Patti L. Ephraim², Iris Pounds', Felicia Hill-Briggs ${ }^{3}$, Linda Darrell ${ }^{4}$, Matthew Ellis ${ }^{5}$, Debra Sudan ${ }^{6}$,
} Hamid Rabb ${ }^{7}$, Dorry Segev ${ }^{8}$, Nae-Yuh Wang ${ }^{3}$, Mary Kaiser ${ }^{8}$, Margaret Falkovic ${ }^{1}$, Jill F. Lebov ${ }^{1}$ and

L. Ebony Boulware ${ }^{1 *}$

\begin{abstract}
Background: Live donor kidney transplantation (LDKT), an optimal therapy for many patients with end-stage kidney disease, is underutilized, particularly by African Americans. Potential recipient difficulties initiating and sustaining conversations about LDKT, identifying willing and medically eligible donors, and potential donors' logistical and financial hurdles have been cited as potential contributors to race disparities in LDKT. Few interventions specifically targeting these factors have been tested.
\end{abstract}

Methods/Design: We report the protocol of the Talking about Living Kidney Donation Support (TALKS) study, a study designed to evaluate the effectiveness of behavioral, educational and financial assistance interventions to improve access to LDKT among African Americans on the deceased donor kidney transplant recipient waiting list. We adapted a previously tested educational and social worker intervention shown to improve consideration and pursuit of LDKT among patients and their family members for its use among patients on the kidney transplant waiting list. We also developed a financial assistance intervention to help potential donors overcome logistical and financial challenges they might face during the pursuit of live kidney donation. We will evaluate the effectiveness of these interventions by conducting a randomized controlled trial in which patients on the deceased donor waiting list receive 1) usual care while on the transplant waiting list, 2) the educational and social worker intervention, or 3) the educational and social worker intervention plus the option of participating in the financial assistance program. The primary outcome of the randomized controlled trial will measure potential recipients' live kidney donor activation (a composite rate of live donor inquiries, completed new live donor evaluations, or live kidney donation) at 1 year.

Discussion: The TALKS study will rigorously assess the effectiveness of promising interventions to reduce race disparities in LDKT.

Trial registration: NCT02369354.

Keywords: Live donor kidney transplant, Live kidney donation, African Americans, Disparities, Randomized clinical trial

\footnotetext{
*Correspondence: ebony.boulware@duke.edu

'Division of General Internal Medicine, Duke University School of Medicine, Durham, NC 27701, USA

Full list of author information is available at the end of the article
} 


\section{Background}

Live donor kidney transplantation (LDKT) represents an optimal therapy for many patients with end-stage kidney disease, and it is associated with substantially improved quality of life and survival compared to dialysis [1-3]. However, African Americans have been persistently and substantially less likely to receive LDKT when compared to Whites [4]. Numerous factors ranging from recipient difficulties initiating and completing transplant evaluations [5-7] to difficulties identifying donors $[8,9]$, and donor difficulties completing medical evaluations have been cited as contributors to race disparities in LDKT [10]. To date, however, few interventions specifically targeting factors that could hinder African Americans from obtaining LDKT have been tested.

The process of obtaining LDKT requires that potential recipients and donors are able to overcome several possible communication and logistical challenges, some of which may affect African Americans disproportionately and contribute to their lower rates of LDKT compared to Whites. For instance, as a critical first step in the process, patients with kidney disease, their families, and their physicians must engage in discussions about live donor kidney transplantation. Patient-physician discussions about LDKT are needed to establish whether LDKT is a viable and/or desirable treatment option for potential recipients, and discussions are needed to ensure that potential recipients understand the risks and benefits of LDKT. Family-physician discussions are also important in helping family members (who not only help potential recipients make informed treatment decisions but who also could serve as potential donors) understand the process of LDKT and associated risks and benefits. Discussions occurring within families about LDKT help families establish whether it is possible to identify willing and medically eligible live donors, and they help families discuss numerous considerations, including the potential psychological, physical, and financial strains of LDKT on family health and priorities. Studies have shown that even when African American patients want to obtain LDKT, they engage in discussions about LDKT with their physicians and their family members at suboptimal rates [11]. African Americans are also less likely than Whites to report they know about transplant prior to initiating dialysis $[12,13]$, and they cite numerous barriers to discussing LDKT, including their discomfort with initiating discussions, concerns about burdening family members, and their uncertainty regarding the appropriate timing for initiating discussions [14]. Once discussions have occurred, patients and their potential donors must consider numerous additional logistical and financial demands placed on potential donors during the LDKT process. For instance, potential donors may require childcare or travel to transplant centers during the evaluation process, and they may also require unpaid time away from work. Evidence suggests African Americans may be more sensitive than Whites to logistical and financial barriers to LDKT [15-17].

African Americans who are on the deceased donor kidney transplant waiting list have already overcome many barriers to seeking kidney transplants [5, 18]. However, communication, logistical and financial barriers may prevent them from pursuing LDKT while waiting for a deceased donor kidney. Evidence suggests interventions employing behavioral support personnel such as transplant social workers to help patients consider LDKT, discuss LDKT with their physicians and families, and overcome logistical barriers to considering or pursuing LDKT could facilitate African Americans' consideration of LDKT $[14,19,20]$. Donor financial assistance programs such as the National Living Donor Assistance Center program (NLDAC) (https:// www.livingdonorassistance.org/default.aspx), [21] could also help African Americans overcome financial barriers to LDKT. However, studies have not been performed to determine whether these interventions can be employed to improve African Americans' access to LDKT.

We describe the protocol for a randomized controlled trial designed to study the effectiveness of behavioral, educational and financial assistance interventions to improve access to LDKT among African Americans on the deceased donor kidney transplant waiting list.

\section{Methods/ Design \\ Overview}

The Talking about Living Kidney Donation Support (TALKS) study is a randomized controlled trial designed to test the incremental effectiveness of (1) a culturally sensitive educational and behavioral social worker intervention and (2) a live donor financial assistance intervention to improve potential kidney recipient activation (i.e., discussions with physicians and family about LDKT) and live kidney donation among African American patients on the deceased donor kidney transplant waiting list at Duke University Medical Center. We adapted the previously developed Talking About Live Kidney Donation (TALK) Social Worker protocol [22] for use among transplant patients on the deceased donor kidney waiting list and developed a live donor financial assistance program. These interventions were designed to overcome communication, logistical, and financial barriers to LDKT (Table 1). The protocol was approved by the Duke University School of Medicine Institutional Review Board.

\section{TALK Social Worker Intervention}

The Talking about Live Kidney Donation Social Worker Intervention (TALK SWI) was previously developed to 
Table 1 Proposed mechanisms through which interventions lead to greater pursuit of LDKT and live kidney donation among African Americans on the deceased donor kidney transplant waiting list

\begin{tabular}{|c|c|}
\hline \multirow[t]{2}{*}{ Barrier to LDKT } & Proposed mechanisms \\
\hline & Educational and social worker support \\
\hline \multirow[t]{2}{*}{ Knowledge barriers } & $\begin{array}{l}\text { - Educational video and booklet introduce } \\
\text { patients and their families to LDKT }\end{array}$ \\
\hline & $\begin{array}{l}\text { - Social worker refers patient to health care } \\
\text { professionals able to discuss risks with } \\
\text { patient and potential donors }\end{array}$ \\
\hline \multirow{2}{*}{$\begin{array}{l}\text { Interpersonal difficulties } \\
\text { initiating and sustaining } \\
\text { LDKT discussions with } \\
\text { family, health care team }\end{array}$} & $\begin{array}{l}\text { - Educational video and booklet encourage } \\
\text { LDKT discussions }\end{array}$ \\
\hline & $\begin{array}{l}\text { - Social worker encourages LDKT discussions, } \\
\text { help patients overcome self-identified } \\
\text { barriers to LDKT discussions }\end{array}$ \\
\hline \multirow[t]{2}{*}{$\begin{array}{l}\text { Logistical and financial } \\
\text { barriers }\end{array}$} & $\begin{array}{l}\text { - Social worker provides patients and } \\
\text { families with information on existing } \\
\text { financial resources for recipient and } \\
\text { potential donor }\end{array}$ \\
\hline & $\begin{array}{l}\text { - Social worker offers financial assistance } \\
\text { Intervention to assist with child care or } \\
\text { uncovered donor expenses related to } \\
\text { donor evaluation, donation, and donor } \\
\text { recovery }\end{array}$ \\
\hline
\end{tabular}

${ }^{\mathrm{a}}$ Those randomly assigned to financial assistance intervention only

improve potential recipients' pre-emptive activation toward LDKT among patients with chronic kidney disease who had not yet developed kidney failure [20, 22]. TALK SWI is a culturally sensitive theory-based intervention, developed in collaboration with a behavioral psychologist, transplant nephrologists, transplant surgeons, transplant social workers, patient advocacy experts from the National Kidney Foundation of Maryland, and with the input from patients and families with CKD and LDKT experience. It consists of an educational booklet and video coupled with a social worker-led brief behavioral support intervention to help patients and their families overcome barriers to considering and pursuing LDKT. In a recent randomized controlled trial, the TALK SWI was effective in activating patients to consider and pursue LDKT, including their increased engagement in LDKT discussions, their completion of LDKT medical evaluations, and their identification of potential live donors [19]. TALK SWI directly addresses many concerns previously identified among African American patients and families considering LDKT [14] (Table 1).

TALK SWI education The TALK SWI educational component consists of a 20-min video and booklet that are designed for patients and their families to review alone or together prior to a visit with the TALK Social Worker. The video features minority and non-minority patients who have undergone LDKT and their family members discussing their experiences with considering
LDKT as a treatment option from the recipient and donor perspectives, as well as health care providers (transplant surgeon, transplant nephrologist, transplant social worker) citing key factors patients and families should consider when contemplating LDKT. It also directly addresses concerns that may pose specific barriers to LDKT for African Americans, including mistrust or fear of the LDKT process, difficulties discussing LDKT, and financial considerations related to pursuing LDKT (Table 2). The educational booklet provides a synopsis of the LDKT process from recipient and donor perspectives. It also includes a listing of publicly available resources from which further information about the LDKT evaluation, transplant, and donation process, clinical risks with LDKT, and financial issues related to LDKT can be obtained. To assist patients and family members with initiating LDKT discussions or addressing complex issues during LDKT discussions (e.g., donor coercion), the booklet also presents several 'model conversations' presenting examples on how to initiate and sustain LDKT discussions. The TALK SWI booklet and video were screened to ensure their appropriateness for all persons considering LDKT, including minority and non-minority persons with low (i.e. 4th grade) reading level and low health literacy.

TALK SWI behavioral support The behavioral support component is a protocol-driven individual and familybased social intervention applying a Social Constructionbased Family Problem Solving theoretical framework [23-25]. According to this framework, families are problem solving units whose optimal structure for confronting problems potentially affecting all group members, such as ESRD and LDKT, is achieved when a neutral authority figure is designated as the mediator for relaying messages between all members and encouraging open channels of communication to enable each member to contribute to the problem's resolution, ultimately enhancing group satisfaction. The protocol specifies that the

Table 2 Barriers to LDKT identified ${ }^{a}$ by patients and family members considering LDKT and addressed by TALK SWI

\begin{tabular}{ll}
\hline Patients & Families \\
\hline Difficulty initiating discussions on own & $\begin{array}{l}\text { Feeling overwhelmed by } \\
\text { patients' disease }\end{array}$ \\
$\begin{array}{l}\text { Concern about being misinterpreted } \\
\text { during LDKT discussions }\end{array}$ & $\begin{array}{l}\text { Patients' denial as barrier to } \\
\text { discussions }\end{array}$ \\
$\begin{array}{l}\text { Concern about burdening family } \\
\text { members }\end{array}$ & Caregiver stress \\
Concern about guilt/ potential & Uncertainty about their own \\
donor coercion & health risks \\
Financial Concerns & Financial Concerns \\
\hline
\end{tabular}

${ }^{a}$ Identified in focus groups of African American and non-African American patients with CKD [14] 
TALK Social Worker will meet with patient participants considering LDKT during two visits. During the first visit, the Social Worker will meet individually with the patient participant for up to one hour to assess their perceived barriers to completing key behaviors reflecting their consideration and pursuit of LDKT (discussing LDKT with their families, discussing LDKT with their physicians, identifying a potential live donor). The TALK Social Worker employs motivational interviewing techniques to help patients self-identify potential barriers they face toward completing LDKT and to help patients strategize about ways they might overcome barriers. At the time of the first visit, the TALK Social Worker invites patients to bring adult family members and/or friends (henceforth shortened to "family members") for a second visit. During the second visit, the TALK Social Worker assesses the extent to which previous discussions with family members about patients' kidney disease had occurred, the results of such conversations, whether family members have communicated about LDKT with patients' physicians, and any barriers family members perceive toward achieving LDKT [20, 22].

Intervention adaptation We reviewed the original TALK SWI protocol with transplant personnel, including a social worker, at the Duke University Kidney and Pancreas Transplant Program and adapted the TALK SWI into their existing workflows. Adaptation focused on (1) ensuring the intervention adequately addresses patients' communication and financial needs in social workers' views, and (2) ensuring the intervention can be feasibly implemented and sustained by transplant centers long-term. For instance, we asked social workers and coordinators to identify the resources they typically provide to patients and families concerned about the financial aspects of LDKT and we incorporated usual work flows regarding financial support into the protocol. Further, in our prior focus groups of patients and families considering LDKT or with LDKT experience, groups identified additional support roles that transplant social workers would be well qualified to fill, including a role as a point of contact for patients and families seeking financial assistance with LDKT [14]. We refined the original TALK SWI behavioral support protocol to include these additional roles that are routinely filled by social workers in transplant centers. We also asked transplant program social workers, nurse coordinators, administrators, and transplant surgeons and nephrologists about clinical workflows entailed with recipient and donor evaluations in an effort to integrate interventions to complement existing clinical programs.

\section{Live donor financial assistance intervention}

We have designed a financial assistance intervention to provide support for potential living kidney donors' medical and non-medical expenses associated with pursuing live kidney donation. The intervention is modeled after the National Living Donor Assistance Center (NLDAC), a federally funded program administered by the Division of Transplantation (DoT), Healthcare Systems Bureau (HSB), Health Resources and Services Administration (HRSA), United States Health and Human Services (HHS) through a cooperative agreement with the University of Michigan and the American Society of Transplant Surgeons (http://www.livingdonorassistance.org/ default.aspx) [21]. It is intended to provide financial support for potential live kidney donors in circumstances where existing federal programs do not provide support. We will offer patient participants the option of enrolling their willing potential live kidney donors (i.e. persons who may wish to pursue live kidney donation) in the financial assistance program, which will provide reimbursement for medical and non-medical expenses related to the evaluation, surgery, and recovery periods associated with live kidney donation. Each patient participant (potential kidney recipient) will be offered a "bank" of $\$ 2100.00$ from which potential donors can receive reimbursement for live kidney donation related expenses. While multiple people may step forward to be evaluated for donation and incur expenses, the total amount available per patient participant is $\$ 2100.00$. Our intervention will provide reimbursement to a broad group of potential donors, of which many may not meet NLDAC income requirements. Participants not qualifying for NLDAC may not require the same level of financial reimbursement, or those choosing to forgo NLDAC may desire more broadly applicable financial assistance (e.g., for lost wages from work) than assistance provided by NLDAC (Table 3). Based on national data in 2010, $\$ 2100.00$ corresponded to 3 weeks of paid leave from work for production or non-supervisory workers [26], approximately 4 weeks of child care, [27] or travel and lodging needs for donors coming from other geographic areas. If effective, our intervention may provide rationale for expanding current qualification requirements for programs such as NLDAC.

We will reimburse potential donors for approved financial assistance through the study by submitting formal original invoices, receipts, and other documentation of their need for reimbursement of qualified medical and non-medical expenses related to live kidney donation evaluation, donation, or convalescence (up to one year after the patient participant enrolls in the study). Qualifying expenses include travel, lodging, meals, incidental expenses (e.g., parking, long-distance phone calls), lost 
Table 3 Live donor financial assistance intervention details and qualifying expenses

\begin{tabular}{lll}
\hline Feature & Proposed intervention & NLDAC \\
\hline Financial assistance amount & $\$ 2100$ & $\$ 6000$ \\
Potential donor and recipient income limits & No & $300 \%$ poverty level or less \\
Proof of donor financial hardship required & No & Yes \\
Covers travel, hotel, parking and meal costs related to donor evaluation, surgery, and follow-up & Yes & Yes \\
Covers lost wages from work related to donor evaluation, surgery, and follow-up & Yes & No \\
Covers child care related costs related to donor evaluation, surgery, and follow-up & Yes & No \\
\hline
\end{tabular}

wages, doctors and hospital visits, and childcare costs incurred by the potential donor as part of (1) donor evaluation, clinic visit or hospitalization, (2) hospitalization for the living donor surgical procedure, and/or (3) medical or surgical follow-up clinic visit or hospitalization within one year of the patient participant's enrollment in the study. Participants will be permitted to utilize the $\$ 2100.00$ for multiple approved purposes, as long as the total value is not greater than $\$ 2100.00$. Furthermore, more than one potential donor may draw from these funds, as the total value of reimbursed expenses does not exceed the value of $\$ 2100.00$ per patient participant. We developed the Live Donor Financial Assistance Intervention following guidelines of the "Organ Donation and Recovery Improvement Act (ODRIA)," (Section 3, 42 U.S.C. 274f) signed into law on April 5, 2004, establishing the authority and legislative parameters to provide reimbursement for travel and subsistence expenses incurred towards living organ donation. The financial assistance program being offered as part of this study is intended to provide reimbursement only in those circumstances when payment cannot reasonably be covered by other sources of reimbursement, including: any State compensation program, an insurance policy, or any Federal or State health benefits program; an entity that provides health services on a prepaid basis; or the recipient of the organ. In order to be reimbursed for travel and qualifying expenses, potential living organ donors must also meet all of the following eligibility criteria and attest to: be a U.S. citizen or U.S. resident; have primary residence in the U.S. or its territories; travel is originating from the donor's primary residence; donor and recipient attest to full compliance with section 301 of the National Organ Transplant Act (NOTA), as amended (42 U.S.C. 274e) which stipulated in part "...(i)t shall be unlawful for any person to knowingly acquire, receive, or otherwise transfer any human organ for valuable consideration for use in human transplantation if the transfer affects interstate commerce"; the transplant center where the donation procedure occurs attests to its status of good standing with the Organ Procurement and Transplantation Network. Additionally, potential donors are eligible for reimbursement for qualifying expenses incurred toward the intended donation of an organ, even if the donation does not occur.

\section{Randomized controlled trial}

We will conduct a randomized controlled trial to assess the effectiveness of TALK SWI and the TALK SWI plus financial assistance intervention to improve rates of LDKT among African American potential transplant recipients on the deceased donor kidney waiting list at the Duke Kidney and Pancreas Transplant Program compared to usual care (Fig. 1). We will follow potential recipients for 1 year to assess live kidney donor activation on potential recipients' behalves (live donor inquiries, completed new live donor evaluations, or live kidney donation) (primary outcome). We will also assess potential recipients' self-reported behaviors reflecting their pursuit of LDKT, including their conduct of LDKT discussions

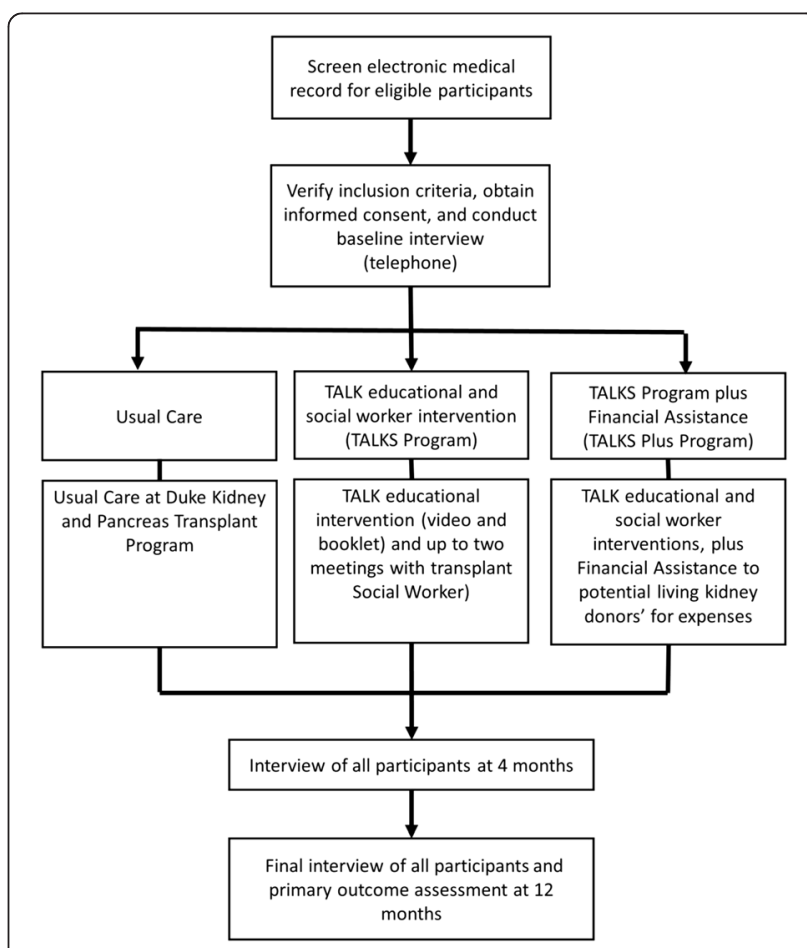

Fig. 1 Overview of study design and randomized controlled trial 
with physicians and with their families and their identification of potential live donors.

Target population We will target adult African American patients with end stage kidney disease who are listed on the deceased donor kidney waiting list of the Duke Kidney and Pancreas Transplant Program. The Duke Kidney and Pancreas Transplant Program cares for a highly diverse set of patients with end stage kidney disease, with nearly $55 \%$ of the deceased donor kidney transplant waiting list comprised of African Americans annually. African Americans on the deceased donor kidney waiting list at Duke Kidney and Pancreas Transplant Program reflect the local Durham, NC population in which $19 \%$ have incomes below the Federal Poverty Level [28]. The Program currently does not have a social worker program tailored specifically to address communication, logistical or financial barriers to LDKT in African American potential recipients.

Screening, eligibility, and recruitment We will enroll qualifying patients ('patient participants') receiving care at the Duke Kidney and Pancreas Transplant Program, their family members and/or friends ('family member participants'), and their potential donors ('potential donor participants') into the study. To identify patient participants, we will screen Duke electronic medical records to identify African American patients who are listed on the deceased donor waiting list at the Duke Kidney and Pancreas Transplant Program with no prior kidney transplant, and who are aged 18 and older. We will obtain appropriate permissions that protect patients' privacy (i.e., HIPAA waiver) prior to all study procedures. We will mail patients who are potentially eligible to participate a recruitment letter signed by all physicians and surgeons in the Duke Kidney and Pancreas Transplant Program, accompanied by information describing the study. This letter will provide instructions for potential participants to call or email the research team with interest in participating or to opt-out from participation by either returning the provided letter in the self-addressed mailed envelope, or by calling the telephone number provided or emailing study staff prior to the first telephone call. Following a 14-day opt-out period, study staff will contact potential patient participants via telephone to confirm their eligibility for participation in the study, answer any questions, obtain verbal consent, and complete a baseline questionnaire. Random assignment to intervention arms will take place immediately following completion of the baseline questionnaire while consenting patient participants are on the telephone.

Family members or friends of enrolled patient participants (i.e. 'family member participants') who attend a social worker visit with patient participants will be asked to provide written consent to participate in the study at the time they present for the social worker visit. Any adult family member or friend who attends will be asked to provide written consent. Potential donor participants interested in the Live Donor Financial Assistance Intervention will only become aware of the intervention through referrals by enrolled patient participants who are randomly assigned to TALK SWI plus financial assistance study arm (see below), who voluntarily choose to make potential donors aware on their own accord, without prompting from study staff or the social worker. Potential donors will contact TALKS Study staff if they are interested in learning more about or want to take part in the TALKS Financial Assistance Intervention. At that time, study staff will address any questions potential donors may have about the intervention. If they decide to participate, study staff will obtain verbal consent over the phone.

\section{Random intervention assignment}

Using blind and secure allocation by computer, we will randomly assign participants to one of three intervention arms: 1) control group (“Usual Care study arm”); 2) TALK SWI ("TALKS Program study arm"); and 3) TALK SWI plus the Live Donor Financial Assistance Intervention ("TALKS Plus Program study arm"). We will perform outcome and correlate assessments at baseline, 4 months, and 12 months for all patient participants following their enrollment in the study (Fig. 1).

Usual care Patient participants assigned to receive Usual Care will receive care as they usually would through the Duke Kidney and Pancreas Transplant Program. In the Usual Care program, all patients meet with a financial coordinator to review financial programs that may be available to them. They also meet with a social worker to discuss transplantation options, social support, and caregiver identification, and to address any questions patients have about transplantation. Through Usual Care, potential donors have the option of meeting with an external Independent Living Donor Advocate, to address any questions or concerns they have about the donation process.

Intervention groups Study staff will mail the TALK video and booklet to patient participants randomly assigned to the TALKS Program and TALKS Plus Program study arms. A letter accompanying the video and booklet will encourage patient participants to review the 20 -min TALK video and booklet and to attend meetings with a study social worker to receive the TALK SWI. The letter will also encourage patient participants to share materials with family members or friends over the age of 18, if they desire. We will invite patient 
participants assigned to the TALKS Program and TALKS Plus Program study arms to meet with a study social worker for up to an hour to discuss their self-identified barriers to LDKT and strategies to overcome barriers. Following this initial meeting, the social worker will invite patient participants to participate in a second session to discuss possible pursuit of LDKT by themselves or with one or more adult family members or friends. TALK social worker visits occur outside of the usual care setting.

If patient participants are randomly assigned to the TALKS Plus Program study arm, study staff will also mail information about the live donor financial assistance intervention along with the TALK video and booklet. Study staff will review the TALKS Study Financial Assistance Intervention with patient participants assigned to this arm at their first visit with the study social worker.

\section{Participant reimbursements}

Patient participants will receive $\$ 30$ for completing each of the questionnaires and $\$ 50$ for each completed session with the social worker. Family participants attending social worker meetings will receive $\$ 20$ total for completing the questionnaire and for participating in the social worker session. Potential donors identified by patient participants in the TALKS Plus Program study arm who want to participate in the Live Donor Financial Assistance Intervention will be asked to complete a Live Donor Financial Assistance Intervention Donor Worksheet summarizing expenses as well as sign a Donor Attestation Form to confirm that they understand the legal restrictions of the intervention. Study staff will mail potential donors checks reimbursing approved eligible expenses.

\section{Data collection and outcomes}

Participant characteristics, correlates of LDKT, and social worker meeting content We will collect information on participant demographics (e.g., age, race, education and income), attitudes/perceptions (e.g., trust in health care team, satisfaction with transplant care), selfreported medical history, and other correlates of LDKT (e.g., health literacy, family function, and self-efficacy with regard to decision-making about LDKT) via telephone questionnaires performed at enrollment, 4 months, and 12 months follow up. In addition to these correlates, we will assess patient participants' perceptions of the cultural competency of the DKPTP and transplant social workers using the Agency for Health Care Research and Quality CAHPS Cultural Competence Item Set [29], designed to capture patients' perspectives on the cultural competence of health care providers. Items include information on patient participants' (a) perceived communication with their transplant health care providers including shared decision-making; (b) experiences of discrimination due to race, ethnicity, insurance, or language; (c) experiences leading to trust or distrust; (d) and linguistic competency (Table 4).

In telephone questionnaires administered at 4 months and 12 months follow up, we will ask patient participants randomly assigned to receive TALKS interventions whether they have reviewed the TALK video and booklet and/or shared them with family members. We will also ask patient participants about their perceptions of the video and booklet. We will ask patient and family member participants who attend the social worker meetings their perceptions of the usefulness of these meetings at meeting conclusion via written questionnaire. With participant consent, we will audio record all social worker meetings to assess content and fidelity to the intervention. Social workers will also take notes to document the content of social worker meetings in electronic case report forms. For patient participants randomly assigned to the TALKS Plus Program study arm, we will assess via questionnaire whether patient participants made others (e.g., family members or friends) aware of their participation in the Live Donor Financial Assistance Intervention. For potential donor participants, we will assess the types of expenditures for which they request reimbursement (Table 4).

Primary and secondary outcomes For our primary outcome, we will assess "live kidney donor activation", defined as the composite rate of live kidney donor inquiries on behalf of patient participants, completed live kidney donor transplant evaluations, and live kidney donor transplants in each arm, ascertained via medical records maintained by the Duke Kidney and Pancreas Transplant Program. Secondarily, we will measure patient participants' behaviors reflecting their interest and pursuit of LDKT, including: self-reported LDKT discussions with physicians, self-reported LDKT discussions with family, and identification of a potential live donor. We will also record if and when deceased donor transplant occurs.

\section{Analysis}

Our primary analyses will test whether the TALKS Program is more effective than usual care and whether TALKS plus the Financial Assistance Intervention (TALKS Plus Program) has additional incremental effectiveness compared to the TALKS Program only. We will assess outcomes at 4 months and 12 months after the intervention, enhancing opportunities to ascertain patient participants' achievement of main outcomes in the event of patient participant attrition or missing data due to missed study visits (e.g., due to hospitalization with ESRD related illness). 
Table 4 Study outcomes and assessments

Baseline

4 months

12 months

Primary outcome: Live kidney donor activation (Composite)

Live donor kidney transplantation (LDKT)

Completed live donor evaluations

Live donor inquiries to transplant center

Secondary outcomes

LDKT discussions with physician

LDKT discussions with family and/or friends

Identification of potential live donor

Belief \& knowledge about treatment for kidney failure, interest in LDKT

Beliefs about treatment for kidney failure

Knowledge of LDKT

Interest in and concerns about LDKT

Knowledge of kidney transplant financial assistance programs

Barriers to and quality of family discussion about LDKT

Mediators and correlates of pursuit of LDKT

Current treatment information

$x$

$x$

$x$

$x$

$x$

$x$

$x$

$x$

$x$

$x$

Socio-demographic Information

Family wealth ${ }^{a}$

Family function (Family APGAR Scale) [32]

Decision self-efficacy about LDKT [33]

Decisional conflict scale [34]

Trust in medical care $[35,36]$

Barriers to obtaining information about LDKT

Depressed mood (PRIME-MD) [37]

Social health (PROMIS-SF) [38, 39]

Risk numeracy [40, 41]

Rapid Estimate of Adult Literacy in Medicine (REALM) [42]

Personal financial well-being scale ${ }^{a}$ [43]

Patient assessment of providers and systems

Cultural competence of health care providers [29]

In-center dialysis care [44]

Nephrologists' communication and caring

Fidelity to TALK SWI protocol, intervention uptake and satisfaction

Participant use of TALK SWI educational materials

Participant satisfaction with TALK SWI sessions ${ }^{a}$

Transplant social worker adherence to TALK SWI protocol

Use of financial assistance programs (by donors) and types of expenditures reimbursed

$x$

$x$

$x$

$x$

$x$

$x$

$x$

$\begin{array}{ccc} & & \\ x & x & x \\ x & x & x\end{array}$

$X$

$x$

$x$

$x+x$

$x \quad x$

$x \quad x$

$x \quad x$

$x \quad x$

$x \quad x$

$x \quad x$

$x \quad x \quad x$

$x \quad x \quad x$

$x \quad x \quad x$

$x \quad x \quad x \quad x$

$x+x$

$x \quad x \quad x$

$x \quad x \quad x$

$\begin{array}{lll}x & x & x\end{array}$

$x \quad x \quad x$

$x \quad x \quad x \quad x$

Information is provided by patient participants and family member participants

The primary outcome is live kidney donor activation (i.e., live donor inquiries to transplantation, completed live donor evaluations, or live donor kidney transplantation). The primary testing contrasts are (1) TALKS Program versus Usual Care, and (2) TALKS Plus Program versus TALKS Program. In secondary analyses, we will test for a trend in effectiveness (TALKS Plus Program more effective than TALKS Program, which is in turn more effective than Usual Care). For the primary testing contrasts, randomly assigned intervention group (i.e., Usual Care, TALKS Program, TALKS Plus Program) will be the main independent variable. For each analysis we 
will use chi-square statistics to assess the proportion of participants in each group achieving at least one live donor activation outcome over 12 months. In the circumstance that there is imbalance in baseline characteristics between the two groups, we will use multivariable analyses to assess differences in live donor activation (dependent variable) over 12 months accounting for group differences. We will account for multiple comparisons (2 primary comparisons). We will assess the prevalence of missing data and examine robustness of intervention effects under various assumptions regarding plausible patterns of missing data in sensitivity analyses.

\section{Sample size estimates}

We will enroll and randomly assign 100 patient participants per study arm. Few randomized controlled trials have been previously performed to establish the effectiveness of interventions to improve rates of live kidney donation and LDKT among African Americans. In our own TALK study among chronic kidney disease patients, we identified a $28 \%$ improvement in achievement of LDKT consideration/pursuit behaviors at 6 months. In another randomized controlled trial, home visits to African American families led to a $20 \%$ improvement in live donor inquiries [30]. Based on these studies, we estimate the TALKS interventions will yield a $25 \%$ improvement in live donor activation behaviors. We are not aware of studies directly studying the effect of financial interventions on live donor activation. We expect that potentially willing donors will be enthusiastic about participating in this intervention and estimate at least an additional $20 \%$ incremental increase in live donor activation with the financial assistance intervention. Under usual circumstances, we estimate approximately $25 \%$ of African American patients in the Duke Kidney and Pancreas Transplant Program deceased donor transplant waiting list receive inquiries from live donors interested in being evaluated on waiting list registrants' behalves each year. Under these assumptions, we estimate we will have approximately $95 \%$ power to detect a $23-30 \%$ difference between TALKS Program study arm and Usual Care study arm at follow up as well as a $21-25 \%$ difference between TALKS Plus Program study arm and the TALKS Program study arm at follow-up. We also estimate we will have $99 \%$ power to observe a trend across the intervention arms, while accounting for multiple comparisons, and accounting for $80 \%$ attrition of participants.

\section{Discussion}

Interventions to improve African Americans' access to LDKT are sorely needed, as trends indicate few reductions in disparities in receipt of LDKT have been made over the past 10 years [31]. African American patients on the live donor kidney transplant waiting list are likely to be highly motivated to receive transplants and have already overcome some barriers to receiving a transplant by completing their recipient transplant evaluations. Nonetheless, they may not have completed key steps to receiving LDKT, contributing to disparities in their access to this optimal treatment. TALK interventions are designed to be culturally sensitive and to directly target barriers to LDKT previously identified as important to African Americans, but they have not yet been tested among African American patients on the deceased donor kidney waiting list. The Live Donor Financial Assistance Intervention extends TALK interventions by additionally addressing potential donor logistical and financial barriers to LDKT.

To our knowledge, TALKS will be one of the first National Institutes of Health (NIH) funded studies to evaluate the effectiveness of combining educational and behavioral interventions with live donor financial assistance to help African Americans on the waiting list receive LDKT. In addition to providing valuable information on the incremental effectiveness of the live donor financial assistance intervention to improve access to LDKT, information we obtain on the types of expenditures potential donors seek for reimbursement will broaden our understanding of the types of assistance which potential donors need the most. Evidence on the uptake and effectiveness of the financial assistance intervention will help inform current national and regional program resource allocation as well as the need and/or feasibility of expanding current programs.

As one of few clinical trials in the field, the TALKS Study will provide needed rigorous evidence to identify practical and effective strategies to improve disparities in LDKT. If TALKS interventions are effective, their ultimate translation into clinical practice changes will be required to yield substantive and lasting improvements in LDKT rates among African Americans.

\section{Abbreviations \\ ESRD: End stage renal disease; LDKT: Live donor kidney transplantation; TALKS: Talking about Living Kidney Donation Support; TALK SWI: Talking about living kidney donation social worker intervention; NLDAC: National Living Donor Assistance Center; DoT: Division of Transplantation; HSB: Healthcare Systems Bureau; HRSA: Health Resources and Services Administration; HHS: Health and Human Services; ODRIA: Organ Donation and Recovery Improvement Act; NOTA: National Organ Transplant Act.}

Competing interests

The authors declare that they have no competing interests.

\section{Authors' contributions}

TSS, PLE, FH-B, IP, ME, DS, HR, and NW contributed to the study design, intervention development, study conduct, and drafting and approval of the manuscript. JFL contributed to the drafting of the manuscript. LEB led the study conception and design, intervention development, study conduct, and drafting and approval of the manuscript. All authors read and approved the final manuscript. 
Authors' information

Not applicable

\section{Availability of data and materials}

Not applicable

\section{Acknowledgements}

The Authors would like to thank Rebekah Wood, MSW for her contributions to the intervention adaption phase.

\section{Funding}

Funding is from the National Institutes of Diabetes and Digestive and Kidney Diseases (NIDDK) 1R01DK098759.

\section{Author details}

${ }^{1}$ Division of General Internal Medicine, Duke University School of Medicine, Durham, NC 27701, USA. ²Department of Epidemiology, Johns Hopkins Bloomberg School of Public Health and Welch Center for Prevention, Epidemiology and Clinical Research, Johns Hopkins Medical Institutions, Baltimore, MD, USA. ${ }^{3}$ Welch Center for Prevention, Epidemiology and Clinical Research, Johns Hopkins Medical Institution and Division of General Internal Medicine, Johns Hopkins School of Medicine, Baltimore, MD, USA. ${ }^{4}$ Department of Social Work, Morgan State University, Baltimore, MD, USA ${ }^{5}$ Division of Nephrology, Duke University School of Medicine, Durham, NC, USA. ${ }^{6}$ Division of Transplantation, Duke University School of Medicine, Durham, NC, USA. ${ }^{7}$ Division of Nephrology, Johns Hopkins School of Medicine, Baltimore, MD, USA. ${ }^{8}$ Division of Transplantation, Johns Hopkins School of Medicine, Baltimore, MD, USA.

\section{Received: 14 July 2015 Accepted: 22 September 2015}

Published online: 09 October 2015

\section{References}

1. Tennankore KK, Kim SJ, Baer HJ, Chan CT. Survival and hospitalization for intensive home hemodialysis compared with kidney transplantation. J Am Soc Nephrol. 2014:25(9):2113-20.

2. Pauly RP, Gill JS, Rose CL, Asad RA, Chery A, Pierratos A, Chan CT. Survival among nocturnal home haemodialysis patients compared to kidney transplant recipients. Nephrol Dial Transplant.2009;24(9):2915-9. doi:10.1093/ ndt/gfp295.

3. Glanton CW, Kao T-C, Cruess D, Agodoa LY, Abbott KC. Impact of rena transplantation on survival in end-stage renal disease patients with elevated body mass index. Kidney Int. 2003;63(2):647-53.

4. Gore JL, Danovitch G, Litwin M, Pham PT, Singer JS. Disparities in the utilization of live donor renal transplantation. Am J Transplant. 2009;9(5):1124-33.

5. Alexander GC, Sehgal AR. Why hemodialysis patients fail to complete the transplantation process. Am J Kidney Dis. 2001;37(2):321-8.

6. Alexander GC, Sehgal AR. Barriers to cadaveric renal transplantation among blacks, women, and the poor. Jama. 1998;280(13):1148-52.

7. Waterman AD, Peipert JD, Hyland SS, McCabe MS, Schenk EA, Liu J. Modifiable patient characteristics and racial disparities in evaluation completion and living donor transplant. Clin J Am Soc Nephrol. 2013;8(6):995-1002.

8. Purnell TS, Powe NR, Troll MU, Wang NY, Haywood Jr C, LaVeist TA, et al. Measuring and explaining racial and ethnic differences in willingness to donate live kidneys in the United States. Clin Transpl. 2013;27(5):673-83.

9. Lunsford SL, Simpson KS, Chavin KD, Menching KJ, Miles LG, Shilling LM et al. Racial disparities in living kidney donation: is there a lack of willing donors or an excess of medically unsuitable candidates? Transplantation. 2006;82(7):876-81.

10. Weng FL, Joffe MM, Feldman HI, Mange KC. Rates of completion of the medica evaluation for renal transplantation. Am J Kidney Dis. 2005:46(4):734-45.

11. Boulware L, Meoni LA, Fink NE, Parekh RS, Kao W, Klag MJ, et al. Preferences, Knowledge, Communication and Patient-Physician Discussion of Living Kidney Transplantation in African American Families. Am J Transplant. 2005;5(6):1503-12.

12. Ayanian JZ, Cleary PD, Weissman JS, Epstein AM. The effect of patients' preferences on racial differences in access to renal transplantation. $\mathrm{N}$ Engl J Med. 1999;341(22):1661-9.

13. Finkelstein FO, Story K, Firanek C, Barre P, Takano T, Soroka S, et al. Perceived knowledge among patients cared for by nephrologists about chronic kidney disease and end-stage renal disease therapies. Kidney Int. 2008:74(9):1178-84

14. Boulware LE, Hill-Briggs F, Kraus ES, Melancon JK, Senga M, Evans KE, et al. Identifying and addressing barriers to African American and non-African American families' discussions about preemptive living related kidney transplantation. Prog Transplant. 2011;21(2):97-104. quiz 105.

15. Ganji S, Ephraim PL, Ameling JM, Purnell TS, Lewis-Boyer LL, Boulware LE. Concerns regarding the financial aspects of kidney transplantation: perspectives of pre-transplant patients and their family members. Clin Transpl. 2014;28(10):1121-30.

16. Gill J, Dong J, Rose C, Johnston O, Landsberg D. The effect of race and income on living kidney donation in the United States. J Am Soc Nephrol. 2013:24(11):1872-9.

17. Lunsford SL, Shilling LM, Chavin KD, Martin MS, Miles LG, Norman ML, et al, Racial differences in the living kidney donation experience and implications for education. Progress in transplantation (Aliso Viejo, Calif). 2007:17(3):234-40.

18. Purnell TS, Hall YN, Boulware LE. Understanding and overcoming barriers to living kidney donation among racial and ethnic minorities in the United States. Adv Chronic Kidney Dis. 2012;19(4):244-51. doi:10.1053/j.ackd.2012.01.008.

19. Boulware LE, Hill-Briggs F, Kraus ES, Melancon JK, Falcone B, Ephraim PL, et al. Effectiveness of educational and social worker interventions to activate patients discussion and pursuit of preemptive living donor kidney transplantation: a randomized controlled trial. Am J Kidney Dis. 2013;61(3):476-86. doi:10.1053/ j.ajkd.2012.08.039. Epub 2012 Oct 22. PubMed PMID: 23089512; PubMed Central PMCID: PMC3710736.

20. DePasquale N, Hill-Briggs F, Darrell $L$, Boyér $L L$, Ephraim $P$, Boulware LE. Feasibility and acceptability of the TALK social worker intervention to improve live kidney transplantation. Health Soc Work. 2012:37(4):234-49.

21. Warren PH, Gifford KA, Hong BA, Merion RM, Ojo AO. Development of the National Living Donor Assistance Center: reducing financial disincentives to living organ donation. Prog Transplant. 2014;24(1):76-81.

22. Boulware LE, Hill-Briggs F, Kraus ES, Melancon JK, McGuire R, Bonhage B, et al. Protocol of a randomized controlled trial of culturally sensitive interventions to improve African Americans' and non-African Americans' early, shared, and informed consideration of live kidney transplantation: the Talking About Live Kidney Donation (TALK) Study. BMC Nephrol. 2011;12:34.

23. Bray JH. Family assessment: Current issues in evaluating families. Fam Relat. 1995:469-477.

24. Cox RP. Theory-based Family Problem Solving Interventions. Clin Excel Nurse Pract. 2002;5(6):3-12

25. Tallman I. The Family as a Small Problem Solving Group. Journal of Marriage and Family. 1970;30(1):94-104.

26. U.S. Department of Labor. National Compensation Survey December 2009 - Jan 2011. In.: Bureau of Labor Statistics; http://uww.bls.gov/ncs/ncswage2010.pdf.

27. U.S. Department of Agriculture. Expenditures on Children by Families, 2010. In.; 2011: http://www.cnpp.usda.gov/sites/default/files/expenditures_on_ children by families/CRC2010.pdf.

28. U.S. Census Bureau: State and County QuickFacts. Data derived from Population Estimates ACS, Census of Population and Housing, State and County Housing Unit Estimates, County Business Patterns, Nonemployer Statistics, Economic Census, Survey of Business Owners, Building Permits In., Last revised February 5, 2015 edn.

29. CAHPS Cultural Competence Item Set. [http://www.cahps.ahrq.gov/]

30. Rodrigue J, Cornell D, Lin J, Kaplan B, Howard R. Increasing Live Donor Kidney Transplantation: A Randomized Controlled Trial of a Home-Based Educational Intervention. Am J Transplant. 2007:7(2):394-401.

31. Matas AJ, Smith JM, Skeans MA, Thompson B, Gustafson SK, Stewart DE, et al. OPTN/SRTR 2013 Annual Data Report: Kidney. Am J Transplant. 2015;15(S2):1-34

32. Good MJ, Smilkstein G, Good BJ, Shaffer T, Arrons T. The family APGAR index: a study of construct validity. J Fam Pract 1979;8:577-82.

33. O'Connor A. User manual-decision self-efficacy scale. Patient Decision Aids, Ottawa Hospital Research Institute (OHIR) Web site https://decisionaid.ohri.ca/ docs/develop/Tools/Decision_SelfEfficacy.pdf (accessed 4 Feb 2015) 1995.

34. O'Connor A. User Manual - Decisional Conflict Scale. Patient Decision Aids, Ottawa Hospital Research Institute (OHIR) Web site https://decisionaid.ohri.ca/docs/ develop/User_Manuals/UM_Decisional_Conflict.pdf (accessed 4 Feb 2015) 1993.

35. Corbie-Smith G, Thomas SB, George DMMS. Distrust, race, and research. Arch Intern Med. 2002;162(21):2458-63. 
36. LaVeist TA, Isaac LA, Williams KP. Mistrust of health care organizations is associated with underutilization of health services. Health Serv Res. 2009;44(6):2093-105.

37. Spitzer RL, Kroenke K, Williams JB, Group PHQPCS. Validation and utility of a self-report version of PRIME-MD: the PHQ primary care study. Jama. 1999;282(18):1737-44.

38. Domain Frameworks. [http://www.nihpromis.org/measures/ domainframework1\#sh.]

39. Hahn E, DeVellis R, Bode R, Garcia S, Castel L, Eisen S, et al. Measuring social health in the patient-reported outcomes measurement information system (PROMIS): item bank development and testing. Qual Life Res. 2010;19(7):1035-44.

40. Fagerlin A, Zikmund-Fisher BJ, Ubel PA, Jankovic A, Derry HA, Smith DM. Measuring numeracy without a math test: development of the Subjective Numeracy Scale. Med Decis Mak. 2007;27(5):672-80.

41. Schapira MM, Davids SL, McAuliffe TL, Nattinger AB. Agreement between scales in the measurement of breast cancer risk perceptions. Risk Anal. 2004;24(3):665-73.

42. Davis TC, Long SW, Jackson RH, Mayeaux E, George RB, Murphy PW, et al. Rapid estimate of adult literacy in medicine: a shortened screening instrument. Fam Med. 1993;25(6):391-5.

43. Prawitz AD, Garman ET, Sorhaindo B, O'Neill B, Kim J, Drentea P. InCharge financial distress/financial well-being scale: Development, administration, and score interpretation. Journal of Financial Counseling and Planning 2006, 17(1)

44. In-Center Hemodialysis CAHPS Survey. https://ichcahps.org/ Default.aspx?tabid=88. Accessed on October 2,2015.

\section{Submit your next manuscript to BioMed Central and take full advantage of:}

- Convenient online submission

- Thorough peer review

- No space constraints or color figure charges

- Immediate publication on acceptance

- Inclusion in PubMed, CAS, Scopus and Google Scholar

- Research which is freely available for redistribution 